\title{
Detection of fungal damaged popcorn using image property covariance features
}

\author{
Onur Yorulmaz $^{\mathrm{a}, *}$, Tom C. Pearson ${ }^{\mathrm{b}}$, A. Enis Çetin ${ }^{\mathrm{a}}$ \\ ${ }^{a}$ Electrical and Electronics Engineering Department, Bilkent University, Ankara 06800, Turkey \\ ${ }^{\mathrm{b}}$ USDA-ARS-CGAHR, 1515 College Avenue, Manhattan, KS 66502, USA
}

\section{A R T I C L E I N F O}

Article history:

Received 5 October 2011

Received in revised form 25 January 2012

Accepted 20 February 2012

\section{Keywords:}

Covariance features

Correlation features

Image processing

Fungus detection on popcorn kernels SVM

\begin{abstract}
A B S T R A C T
Covariance-matrix-based features were applied to the detection of popcorn infected by a fungus that causes a symptom called "blue-eye". This infection of popcorn kernels causes economic losses due to the kernels' poor appearance and the frequently disagreeable flavor of the popped kernels. Images of kernels were obtained to distinguish damaged from undamaged kernels using image-processing techniques. Features for distinguishing blue-eye-damaged from undamaged popcorn kernel images were extracted from covariance matrices computed using various image pixel properties. The covariance matrices were formed using different property vectors that consisted of the image coordinate values, their intensity values and the first and second derivatives of the vertical and horizontal directions of different color channels. Support Vector Machines (SVM) were used for classification purposes. An overall recognition rate of $96.5 \%$ was achieved using these covariance based features. Relatively low false positive values of $2.4 \%$ were obtained which is important to reduce economic loss due to healthy kernels being discarded as fungal damaged. The image processing method is not computationally expensive so that it could be implemented in real-time sorting systems to separate damaged popcorn or other grains that have textural differences.
\end{abstract}

(C) 2012 Elsevier B.V. All rights reserved.

\section{Introduction}

The drying of grain kernels is an important issue in agriculture that requires precise timing. Grain kernels that are not dried properly may become infected by fungi, thereby greatly reducing the economic value of the product. In popcorn kernels, one of these problematic infections is called blue-eye damage and is caused by fungi from the Penicillium genus. Fungi can spread over the kernels after harvesting if they are not dried rapidly enough. However, popcorn cannot be dried rapidly with the use of high heat because it may crack and be unable to pop. If a balance between the time until storage and the time for proper drying is not achieved successfully, kernels may still be wet when they are sent for binning, thereby creating a favorable environment for fungal infections to spread. Blue-eye damage changes the taste of popped kernels and causes consumers to reject them, reducing the consumption of popcorn and resulting in economic losses for the popcorn industry. Although damaged kernels do not occur with high frequency, one bad kernel can cause a consumer to stop eating the remaining popcorn in a bowl even though the remaining popcorn may not have been infected and do not contain off-flavors. It can also cause the consumer not to buy a particular brand of popcorn.

\footnotetext{
* Corresponding author. Mobile: +90 505483 7450; fax: +90 3122664192.

E-mail address: yorulmazonur@gmail.com (O. Yorulmaz).
}

Blue-eye-infected popcorn kernels have a small blue blemish on the kernels at the center of the germ. This blemish makes it possible to approximate the location of the infection and to detect infected kernels from images taken by regular color cameras. Fig. 1 shows images of undamaged and damaged popcorn kernels that were obtained with a Canon Powershot G11 digital camera.

There have been various studies on the subject of separating blue-eye-damaged and undamaged kernels. Pearson (2009) developed a machine to detect the damaged kernels as they slide down a chute. Three cameras located around the perimeter of the kernel simultaneously obtained images while a Field-Programmable Gate Array (FPGA) processed each image in real time. The array looked for rows in the image matrix in which the intensity values were greater at the borders of the germ and lower in the middle of the germ. The detection of this valley-like shape in image intensity values along a line of the image was used to confirm the existence of blue-eye damage. In this approach, the red channels of the images of the kernels were used, because the kernels are red-yellow, and the damage is more visible in this channel. However, the accuracy of this system, $74 \%$ for the blue-eye damaged popcorn, was not adequate for the system to be useful for the popcorn industry.

Another approach for detecting blue-eye damaged popcorn using cepstral features was proposed by Yorulmaz et al. (2011). Cepstral feature extractions are the most widely used analytical methods in speech processing (Quatieri, 2001). These features are also used for the representation of impact sounds in agricultural 


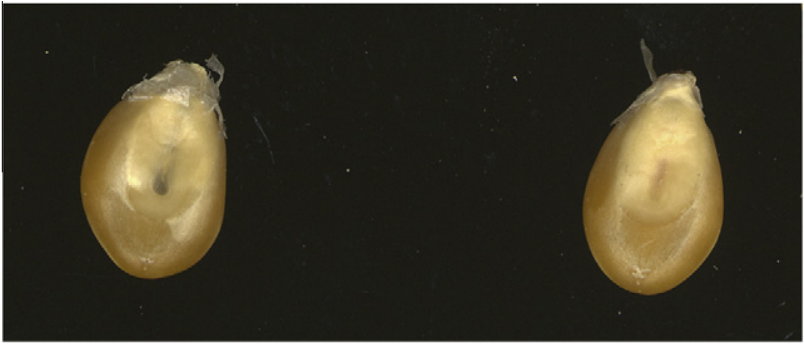

Fig. 1. Images of blue-eye-damaged (left) and undamaged (right) popcorn kernels. (For interpretation of the references to colour in this figure legend, the reader is referred to the web version of this article.)

systems (Cetin et al., 2004; Pearson et al., 2007a,b). Recently, cepstral features have also been used in image representation (Narwaria et al., 2012). A two-dimensional (2D) cepstrum is defined as the 2D inverse Fourier transform of the logarithm of the absolute magnitude of the 2D Fourier transform of an image $x$, as follows:

$\tilde{x}(p, q)=F_{2}^{-1}\left(\log \left(|X(u, v)|^{2}\right)\right)$,

where $X(u, v)$ is the Discrete Fourier Transform (DFT) coefficient matrix of a given image $x, \tilde{x}(p, q)$ is the resulting cepstral features matrix, and $F_{2}^{-1}$ represents the 2D Inverse Discrete Fourier Transform (IDFT) operation.

Cepstral analysis is useful when comparing two similar signals in which one signal is an amplitude-scaled version of the other one. In this case, the two images $x$ and $a x$ have the same cepstrum, except for $x(0,0)$ (Narwaria et al., 2012) because of the logarithmic operation. Because it is based on the magnitude of the Fourier transform, this cepstrum is also shift-invariant. In practice, modified versions of cepstral parameters are used for image representation. This technique has been successfully applied to face recognition (Cakir and Cetin, 2011) and man-made object recognition applications (Eryildirim and Onaran, 2011). The cepstrumbased method in Yorulmaz et al. (2011) groups Fourier coefficients before computing the inverse DFT. In this approach, non-uniform grids are used to reduce the number of cepstral features by combining them. A grid is applied to the Fourier domain coefficients, and the mean values of the magnitudes of the Fourier coefficients inside the bins of the grids are obtained as standalone Fourier values before their logarithms and inverse DFTs are computed. This approach reduces the number of cepstral parameters. In melcepstrum, the bin sizes are smaller and are obtained from low frequencies, rather than from high frequencies because most natural signals and images are low-pass in nature (their signal energy is concentrated in low frequencies). Finally, classification using cepstral features was performed using a Support Vector Machine (SVM).

The objective of this study is to apply image-intensity-based covariance features to the blue-eye detection problem, and to develop a method that can classify damaged and undamaged popcorn kernels in real time. The success rates of this study is compared to the results of the cepstrum-based features from Yorulmaz et al. (2011). Covariance features were proposed and used for object detection by Tuzel et al. (2006). Duman et al. (2009) and Duman and Çetin (2010) applied covariance features to synthetic aperture radar (SAR) images for object detection purposes. Covariance features were introduced as a general solution to object detection problems (Tuzel et al., 2006), where seven image-intensity-based parameters were extracted from the pixels in image frames, and their covariance matrix was used as a feature matrix to represent an image or an image region. The distance between two covariance matrices was computed using generalized eigenvalues in Tuzel et al. (2006). However, this operation is computationally costly. To adapt the algorithm to real-time processing, Duman et al. (2009) and Habiboglu et al. (2011a) used the upper diagonal elements of the covariance matrix as features.

The remainder of the paper is organized as follows: in Section 2 , the intensity-based pixel properties, which are based on Tuzel et al. (2006) and Habiboglu et al. (2011a), and were used to calculate the covariance matrices will be introduced. The covariance feature extraction method used in this paper is also explained in this section. In Section 3, the SVM classification method that was used in this study is presented. The popcorn test set, image acquisition and image pre-treatments will be detailed in Section 4. The experimental results and a comparison with earlier methods for the detection of blue-eye damaged popcorn will be given in Section 5.

\section{Feature extraction from popcorn images}

\subsection{Covariance matrix of intensity and color-based property vectors}

Tuzel et al. (2006) applied covariance feature extraction methods to object detection in videos. Tuna et al. (2009) used this method for image texture classification and forest fire smoke detection in videos. Damaged popcorn kernels have an image texture that is different from that of undamaged kernels. The germ of the kernel has a distinguishing dark or gray region, or simply the darker region in the middle is larger than that of healthy kernels. The exact location of this region changes for each kernel and the orientation. Therefore, it is proposed that a texture classification method can be used to distinguish damaged kernels from undamaged kernels.

The first step is to calculate the property vector of each pixel in the image. Next, the covariance matrix of all property vectors is obtained to represent an image or an image region. Typically, the property vector $\Theta_{i, j}$ of the $(i, j)$ th pixel is composed of gray-scale intensity values or color-based properties and their first and second derivatives. After the property vectors are computed, the covariance matrix of an image is estimated by the following operation:

$\hat{\Sigma}=\frac{1}{N-1} \sum_{i} \sum_{j}\left(\Theta_{i, j}-\bar{\Theta}\right)\left(\Theta_{i, j}-\bar{\Theta}\right)^{T}$,

where $\hat{\Sigma}$ is the estimated covariance matrix; $N$ is the number of pixels in the region; $\Theta_{i, j}$ is the property vector of the pixel located at coordinates $i$ and $j$; and $\bar{\Theta}$ is the mean of $\Theta_{i, j}$ in the given image region, which is calculated as follows:

$\bar{\Theta}=\frac{1}{N} \sum_{i} \sum_{j} \Theta_{i, j}$.

In this study, various property vectors were tested, and the classification performances of different combinations of properties were compared. The gray-scale intensity values may not contribute significantly to the popcorn classification results. However, the property values from separate color channels may improve the recognition rates. As was performed by Tuzel et al. (2006), the color and gray-scale intensity-based properties were combined to build property vectors and were shown to give superior classification results in some applications.

In addition to the red- and blue-channel pixel values, the contributions of the first and second derivative values in the vertical and horizontal directions were tested by including them in the property vectors. The pixel locations were included and excluded in vector definitions to test their contributions to the results. The eight property vectors that were tested are given in Eqs. (4)-(11) as follows: 


$$
\begin{aligned}
& \Theta_{i, j}=\left[R(i, j)\left|\frac{\partial R(i, j)}{\partial x}\left\|\frac{\partial R(i, j)}{\partial y}\right\| \frac{\partial^{2} R(i, j)}{\partial x^{2}} \| \frac{\partial^{2} R(i, j)}{\partial y^{2}}\right|\right]^{T}, \\
& \Theta_{i, j}=\left[R(i, j)\left|\frac{\partial B(i, j)}{\partial x}\left\|\frac{\partial B(i, j)}{\partial y}\right\| \frac{\partial^{2} B(i, j)}{\partial x^{2}} \| \frac{\partial^{2} B(i, j)}{\partial y^{2}}\right|\right]^{T}, \\
& \Theta_{i, j}=\left[R(i, j) B(i, j) \mid \frac{\partial R(i, j)}{\partial x}\left\|\frac{\partial R(i, j)}{\partial y}\right\| \frac{\partial^{2} R(i, j)}{\partial x^{2}} \| \frac{\partial^{2} R(i, j)}{\partial y^{2}}\right]^{T}, \\
& \Theta_{i, j}=\left[R(i, j) B(i, j) \mid \frac{\partial B(i, j)}{\partial x}\left\|\frac{\partial B(i, j)}{\partial y}\right\| \frac{\partial^{2} B(i, j)}{\partial x^{2}} \| \frac{\partial^{2} B(i, j)}{\partial y^{2}}\right]^{T}, \\
& \Theta_{i, j}=\left[i j R(i, j) \mid \frac{\partial R(i, j)}{\partial x}\left\|\frac{\partial R(i, j)}{\partial y}\right\| \frac{\partial^{2} R(i, j)}{\partial x^{2}} \| \frac{\partial^{2} R(i, j)}{\partial y^{2}}\right]^{T}, \\
& \Theta_{i, j}=\left[i j R(i, j) \mid \frac{\partial B(i, j)}{\partial x}\left\|\frac{\partial B(i, j)}{\partial y}\right\| \frac{\partial^{2} B(i, j)}{\partial x^{2}} \| \frac{\partial^{2} B(i, j)}{\partial y^{2}}\right]^{T}, \\
& \Theta_{i, j}=\left[i j R(i, j) B(i, j) \mid \frac{\partial R(i, j)}{\partial x}\left\|\frac{\partial R(i, j)}{\partial y}\right\| \frac{\partial^{2} R(i, j)}{\partial x^{2}} \| \frac{\partial^{2} R(i, j)}{\partial y^{2}}\right]^{T}, \\
& \text { and }
\end{aligned}
$$$$
\Theta_{i, j}=\left[i j R(i, j) B(i, j)\left|\frac{\partial B(i, j)}{\partial x}\left\|\frac{\partial B(i, j)}{\partial y}\right\| \frac{\partial^{2} B(i, j)}{\partial x^{2}} \| \frac{\partial^{2} B(i, j)}{\partial y^{2}}\right|\right]^{T},
$$

where $R(i, j)$ and $B(i, j)$ are, respectively, the red- and blue-channel color values of the pixel located at coordinates $i$ and $j$. For the first and second derivatives, different sizes of derivative filters are tested, and it is observed that the results do not change significantly. Therefore to keep the computation time minimal, derivative filters with small lengths were selected. First and second derivatives of the red-and blue-channel values were calculated by convolution with the [ -101$]$ and [1 -21] filters, respectively; i.e., the image was horizontally (vertically) convolved with the $[-101]$ vector to compute the horizontal (vertical) derivative. The resulting covariance matrices had sizes of $5 \times 5$ for Eqs. (4) and (5), $6 \times 6$ for Eqs. (6) and (7), $7 \times 7$ for Eqs. ( 8 ) and (9), and $8 \times 8$ for Eqs. (10) and (11), respectively.

Coordinate values were also included in the feature vectors (8), (9), (10) and (11) because the blue-eye damage is usually located at the center of the popcorn kernel image. As a result, indexsensitive covariance parameters can be obtained from the feature matrix. Feature vectors (4)-(7) produced location-invariant feature matrices.

\subsection{Covariance and correlation matrix-based image region classification}

The covariance matrices of the image and video regions in either 2D or 3-dimensional spaces can be used as representative features of an object, and they can be compared for classification purposes. As stated in Tuzel et al. (2006), covariance features do not lie in Euclidean space, and, therefore, the distances between covariance matrices cannot be calculated as if they are in Euclidean space. To overcome this problem, Forstner et al. (1999) developed a method based on generalized eigenvalues and used it to measure the similarity of matrices in Tuzel et al. (2006). However, this operation was computationally costly, and because real-time applications require computational efficiency, the elements of the covariance matrices were calculated as if they were feature values in Euclidean space Habiboglu et al. (2011a).

In Habiboglu et al. (2011a), an SVM (Boser et al., 1992) was used as the classifier. The presence of the five elements in the property vectors that were defined in Eqs. (4) and (5) results in $5 \times 5=25$ features in the covariance matrix. Similarly, covariance matrices constructed from the property vectors in Eqs. (6) and (7) had 36 features; Eqs. (8) and (9) produced 49 features, and Eqs. (10) and (11) resulted in a total of 64 features. However, because the covariance matrices are symmetrical with respect to their diagonal elements, only the upper or lower diagonal elements were included in the classification process. The elements of the covariance matrices corresponding to the covariance values of $x y, x x$ and $y y$ (at locations $(1,1),(1,2)$ or $(2,1)$, and $(2,2)$, respectively) were omitted from the covariance matrices that were calculated by Eqs. (8)-(11) because those values do not provide any relevant information about the distributions of intensities. Fig. 2 illustrates the feature selection from covariance matrices that was achieved by Eqs. (4)-(11).

In addition to the covariance matrix features, correlation coefficient descriptors that were defined in Habiboglu et al. (2011b) were also applied to the classification problem. Correlation coefficient-based features were obtained by normalizing the covariance parameters from Eqs. (4)-(11) that were calculated from image pixel values. The correlation coefficient $C(a, b)$ of the $(a, b)$ th entry of the correlation coefficient matrix was calculated as follows:

$C(a, b)=\left\{\begin{array}{c}\sqrt{\Sigma(a, b)}, a=b \\ \frac{\sqrt{\Sigma(a, b)}}{\sqrt{\Sigma(a, a) \Sigma(b, b)}}, \text { otherwise }\end{array}\right.$,

where $\Sigma(a, b)$ is the $(a, b)$ th entry of the covariance matrix $\hat{\Sigma}$ :

$\Sigma(a, b)=\frac{1}{N-1} \sum_{i} \sum_{j} \Theta_{i, j}(a) \Theta_{i, j}(b)-c_{N}(a, b)$,

where $\Theta_{i, j}(a)$ is the property value located in the $a$ th index of the property vector of the pixel with the coordinate values $i$ and $j$, and

$c_{N}(a, b)=\frac{1}{N}\left(\sum_{i} \sum_{j} \Theta_{i, j}(a)\right)\left(\sum_{i} \sum_{j} \Theta_{i, j}(b)\right)$.

\section{Classification}

For both the covariance and correlation methods, the classification of kernels as blue-eye damaged or non-damaged was achieved through an SVM, and the results were compared with those obtained using the mel-cepstrum-based method. The SVM is a supervised classification technique that was developed by Vladimir Vapnik (Boser et al., 1992). The algorithm was implemented as a Matlab library in Chang and Lin (2011) and was also used for this study. The SVM algorithm projects the points in a space into higher-dimensional spaces in which a superior differentiation between classes can be achieved using the Radial Basis Function (RBF) Gaussian kernel. Next, the algorithm finds vectors from the

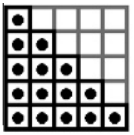

(a)

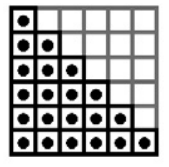

(b)

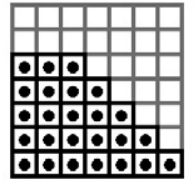

(c)

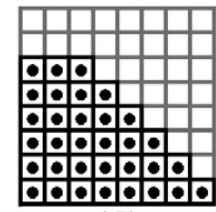

(d)
Fig. 2. Illustration of the feature selection from covariance matrices. Features extracted from the property covariance matrices are shown as black dots: (a) Eqs. (4) and (5), (b) Eqs. (6) and (7), (c) Eqs. (8) and (9), and (d) Eqs. (10) and (11). 
higher-dimensional space that are on the borders of class clouds called support vectors and, using these vectors, classifies the remaining samples. For this work, the RBF kernel of the SVM algorithm was applied. The default parameters of the SVM were used except for the cost (Chang and Lin, 2011). The cost is the parameter that forces the training set to result in more support vectors in order to reduce the training classification mistakes. Very high cost values have a danger of over-fitting. Low cost values may result in under-fitting. In this study, cost value was assigned to be relatively high (500) in order to get better fitted curves in the higher dimensions since we have plenty of training data. As it is a supervised classification method, an SVM must be trained using previously labeled data. For this work, the datasets for training and testing the SVM were randomly divided into 10 subsets of equal size, and with the leave-one out principle, SVM was trained and tested accordingly.

\section{Image acquisition and pre-treatment}

In this study, the image datasets that are tested in Yorulmaz et al. (2011) are used, which included different varieties of popcorn kernels from various years. Samples of popcorn grown in western Iowa from two growing years (2007 and 2008) and from five different storage bins were collected so that a reasonable range of kernel color and kernel morphology could be studied. Samples were drawn from bins known to have high levels ( $\sim 5 \%$ ) of blue-eye damage. Each sample was approximately one kg. Approximately $100 \mathrm{~g}$ from all samples were blended, then divided using a Boerner divider (\#34, Seedburo Co., Des Plaines, IL) until the sample size was reduced to about 500 kernels which were then used for imaging. The varieties were unknown as they were pulled from bins of commercial corn. Most likely, they comprised several varieties. The infesting mold spores were naturally present at the time of harvest, no spores were introduced to the grain as they were loaded into the bins. The ground truth was determined by visual inspection of the popcorn and inspection of the transmittance images. By doing so, there is a danger that slightly infested kernels are not seen, but it is highly unlikely that these would be classified as damaged using the imaging algorithms.

The images of kernels were obtained by placing the kernels in a grid array on a document scanner (Expression 1680, Epson America, Long Beach, CA). Images are taken in two different manners: reflectance and transmittance modes. Reflectance mode images are similar to regular camera images. In this mode, the light reflected from the kernels is captured. In the transmittance mode, the light that passes through the popcorn kernels is captured. The scanner was equipped with a transparency, or negative film attachment to acquire transmittance images. To acquire usable transmittance images of popcorn, the brightness was simply set to the maximum value using the software supplied with the scanner. Single kernels were extracted from the larger original images using a Matlab program. Using this program, a threshold was applied to the red pixels, and the connected pixels that exceeded this threshold were selected as single kernels for the

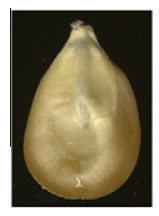

(a)

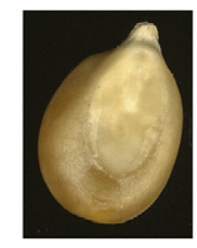

(b)
Fig. 3. (a) Damaged popcorn kernel images acquired in the reflectance (left) and transmittance (right) modes. (b) Undamaged popcorn kernel images acquired in the reflectance (left) and transmittance (right) modes. reflectance-mode images. For the transmittance mode images, the pixels that were below the threshold were selected to be kernel objects. In this dataset, there were 510 undamaged and 398 blueeye damaged popcorn kernel images. It was observed that the damage was more visible in transmittance mode images. Examples of transmittance-mode and reflectance-mode images are shown in Fig. 3.

Because of this process, different sizes and shapes of kernel images were obtained. Typically, the kernel image sizes were 200 by 300 pixels. Because the covariance and correlation features do not depend on the number of pixels, the images do not need to be the same size.

As shown in Fig. 3, the background is included in a typical popcorn kernel image because the image data was obtained in a rectangular manner. To reduce the effects of the background pixel intensities, the approximate location of possible blue-eye damage was cropped from each popcorn image. This operation was performed in proportion to the size of the kernel image. Because blue-eye damage is mostly located in the upper part of a popcorn kernel, the left and right margins were set to be $20 \%$ of the original image width, whereas the top margin was set to $25 \%$ of the image height, and the bottom margin was set at $50 \%$ of the image height, as shown in Fig. 4. Using this approach, a small rectangular region of each image was extracted.

In the popcorn image data sets used in this study, popcorn kernels were oriented manually such that the tips of the germ were on the upper side of the image. However, in real-time sorting applications, popcorn kernels may have different orientations. Therefore, the direction detection algorithm presented by Narwaria et al. (2012) was used to detect the tip of the kernel. For this purpose, the image of each kernel was thresholded and the silhouette of the kernel was determined. The center of the kernel was calculated by taking the mean of threshold passing pixels and the boundary was found by high pass filtering the silhouette image. The high pass filter coefficients do not carry much significance since the image is binary. The distance from the center to the boundary was calculated by spanning $360^{\circ}$ in 64 steps and the second derivative of this distance function was found by the high pass filter $g_{H P}[n]=\left[\begin{array}{lllllll}-1 & 0 & 0 & 2 & 0 & 0 & -1\end{array}\right]$. The maximum value of the absolute value of the second derivative of this distance function was considered to be the tip of the kernel. Therefore, by estimating the tip of the kernel, it was possible to rotate the kernels before the procedure, if they are not oriented manually.

\section{Experimental results}

Covariance and correlation matrix classification results were compared with the Fourier domain cepstral methods developed to identify blue-eye damaged popcorn in Yorulmaz et al. (2011). In that study, cepstrum-based features were also applied to the same transmittance and reflectance mode images used in this study. For the covariance and correlation section of the study, features were extracted using the eight different property vector types that were defined in Eqs. (4)-(11). For comparison with the cepstral features, the property vectors defined in Eqs. (10) and

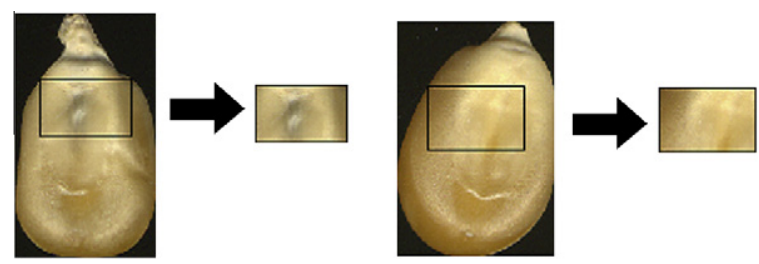

Fig. 4. The cropping operation was performed in proportion to the size of each kernel image. 
(11) were selected from among the covariance and correlation methods to calculate features because they resulted in the best overall recognition rates for both the transmittance and reflectance mode images. As in Yorulmaz et al. (2011), the overall success rates on test set was also calculated by weighting the test results according to the number of test images of two different kernel classes, as shown in Table 1.

In Table 1, the highest recognition rates are highlighted in bold. For transmittance mode images, the correlation features using properties defined in Eq. (11) provided the best overall recognition: $96.5 \%$. The best overall success rate using cepstrum features for this mode was $93.9 \%$, indicating an improvement of $2.5 \%$ in the overall success rate when using the correlation features for this mode. However, the classification accuracies were more uniform for the covariance and correlation methods than for the cepstrum-based feature methods. For example, in the reflectance mode for the mel-cepstrum results, the recognition rates to correctly identify undamaged and damaged kernels varied from $79 \%$ to $86 \%$, whereas those for the covariance- or correlation-based features varied from $89 \%$ to $93 \%$. High recognition in undamaged kernels also indicates low false positive rates which reduces the economic loss by reducing the quantity of healthy kernels being classified as low value fungal damaged kernels.
The best classification accuracy using covariance features, 94\% overall, was observed using reflectance-mode images. Table 1 suggests that the use of covariance features improved the overall success rates of blue-eye damage detection in reflectance mode images by approximately $11 \%$ compared with mel-cepstrum features. This result is important because transmittance-mode images are more difficult to obtain, and it is almost impossible to use this mode in real-time applications. Reflectance-mode imaging, however, is a simpler method and can be achieved using simple cameras and lighting. An overall recognition rate of $94 \%$ on reflectance mode images is important due to this fact.

Another advantage of the covariance method is the higher speed of the algorithm. Although there are fast algorithms for calculating a Fourier transform and its inverse, their usage complicates the performance of real-time applications. However, covariance features are calculated via convolution with small vectors using a small subset of pixels. The filter vector lengths are short for both the first and second derivative calculations, which provide efficient real-time processing using low-cost FPGA hardware. Furthermore, for SVM training and testing, cepstral-based feature vectors have greater lengths. To achieve the best results with cepstral features, a grid with a size of $29 \times 29$ was required, resulting in a feature matrix with 435 values (Yorulmaz et al., 2011). Conversely, covariance

Table 1

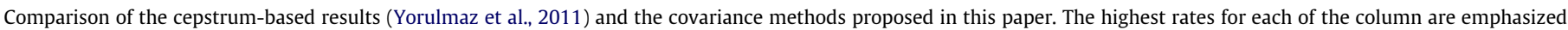
with bold font.

\begin{tabular}{|c|c|c|c|c|c|c|}
\hline & \multicolumn{3}{|c|}{ Reflectance mode success rate (\%) } & \multicolumn{3}{|c|}{ Transmittance mode success rate (\%) } \\
\hline & Overall & Undamaged & Damaged & Overall & Undamaged & Damaged \\
\hline Mel-Cepstrum & 83.1 & 86.3 & 78.9 & 93.9 & 97.4 & 89.4 \\
\hline Correlation Eq. (11) & 91.9 & 93.4 & 89.0 & 96.5 & 97.6 & 95.1 \\
\hline Covariance Eq. (10) & 94.1 & 94.4 & 93.6 & 94.6 & 95.4 & 93.6 \\
\hline
\end{tabular}

Table 2

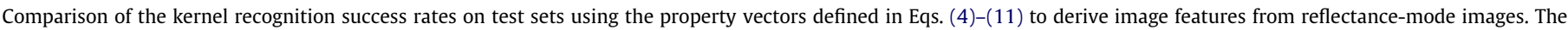
highest rates for each of the column are emphasized with bold font.

\begin{tabular}{|c|c|c|c|c|c|c|}
\hline \multicolumn{7}{|c|}{ Kernel recognition success rate for reflectance mode images } \\
\hline \multicolumn{4}{|c|}{ Covariance features } & \multicolumn{3}{|c|}{ Correlation features } \\
\hline Property vector & Overall & Undamaged & Damaged & Overall & Undamaged & Damaged \\
\hline Eq. (4) & 89.2 & 91.2 & 86.6 & 83.6 & 84.8 & 82.1 \\
\hline Eq. (5) & 85.6 & 87.6 & 83.0 & 81.7 & 87.7 & 74.1 \\
\hline Eq. (6) & 90.4 & 91.9 & 88.5 & 88.1 & 89.7 & 86.1 \\
\hline Eq. (7) & 86.1 & 89.0 & 82.3 & 81.4 & 84.2 & 77.8 \\
\hline Eq. (8) & 92.6 & 93.6 & 91.3 & 92.5 & 93.7 & 91.0 \\
\hline Eq. (9) & 91.9 & 93.9 & 89.3 & 86.8 & 87.8 & 85.6 \\
\hline Eq. (10) & 94.0 & 94.4 & 93.5 & 94.1 & 94.4 & 93.6 \\
\hline Eq. (11) & 91.9 & 93.4 & 99.0 & 88.6 & 88.9 & 88.2 \\
\hline
\end{tabular}

Table 3

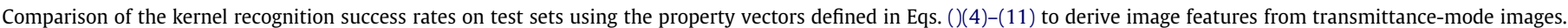
The highest rates for each of the column are emphasized with bold font.

\begin{tabular}{|c|c|c|c|c|c|c|}
\hline \multicolumn{7}{|c|}{ Kernel recognition success rate for transmittance mode images } \\
\hline \multicolumn{4}{|c|}{ Covariance features } & \multicolumn{3}{|c|}{ Correlation features } \\
\hline Property vector & Overall & Undamaged & Damaged & Overall & Undamaged & Damaged \\
\hline Eq. (4) & 92.6 & 94.5 & 90.3 & 92.9 & 95.1 & 90.2 \\
\hline Eq. (5) & 94.7 & 95.1 & 94.2 & 87.6 & 90.0 & 84.5 \\
\hline Eq. (6) & 93.7 & 95.3 & 91.7 & 93.9 & 94.3 & 93.4 \\
\hline Eq. (7) & 96.0 & 97.4 & 94.3 & 89.7 & 91.1 & 87.8 \\
\hline Eq. (8) & 93.9 & 95.6 & 91.8 & 93.7 & 94.9 & 92.2 \\
\hline Eq. (9) & 96.3 & 97.1 & 95.4 & 91.5 & 92.6 & 90.2 \\
\hline Eq. (10) & 96.1 & 96.4 & 95.7 & 94.6 & 95.4 & 93.6 \\
\hline Eq. (11) & 96.5 & 97.6 & 95.1 & 94.3 & 96.5 & 91.6 \\
\hline
\end{tabular}


feature vectors had fewer than 64 values. A reduced number of dimensions results in faster calculations of support vectors, faster decision times for test images and, probably, a more robust classification performance.

A disadvantage of the covariance-based features is that they are not shift-invariant, while cepstrum-based algorithms are. With the use of the absolute value of an FFT, the mel-cepstrum had a translational invariance and added a rotational invariance because of its log-polar conversion. However, the use of the $i$ and $j$ coordinate values causes the covariance method variant to have translational changes. Conversely, shift invariance was achieved in Eqs. (4)-(7), which excluded these properties. Moreover, all of the property vector definitions used for the experiments in this study were rotationally variant because they included the derivative values in the vertical and horizontal directions. Recent advances in kernelhandling mechanisms (Pearson et al., 2011) enabled the rotation of kernels to be constrained; therefore, rotationally variant features are not an overwhelming problem.

A comparison of the results obtained using different property vector definitions is provided in Tables 2 and 3 for reflectanceand transmittance-mode images, respectively. For reflectance mode, overall accuracies ranged from $85.6 \%$ to $94 \%$. While Eq. (10) had the highest overall accuracy, it used 33 property values in the SVM for classification. Eq. (4) had an overall accuracy of $89.2 \%$ but only used 15 property values. Further research on a larger data set is needed to determine the robustness of the property vectors and the selection of the most robust equation. For transmittance images, the range of property vector accuracies was smaller, with values of $92.6-96.5 \%$. The covariance feature property set based on Eq. (5) had an overall accuracy of $94.7 \%$ on the test set and, in accordance with Eq. (4), only consists of 15 covariance values. However, the covariance feature set based on Eq. (11) uses 33 parameters.

Tables 2 and 3 suggest that it is favorable to select different property-vector definitions to calculate covariance and correlation features depending on the image mode. To achieve the best detection rates when using transmittance mode images, Eq. (10) should be selected, and if the images are taken in the reflectance mode, the best results would be achieved with Eq. (11). The only difference between Eqs. (10) and (11) is in the derivative values used in the property vectors. In Eq. (10), the first and second derivatives are calculated using the red channel, whereas in Eq. (11), they are calculated with the blue channel. In addition, the best results when using the reflectance mode were achieved with covariance-based features, while the best results for the transmittance mode were obtained with a correlation method. Therefore, depending on the mode that the images are taken in, the success rates for kernel recognition can be maximized by selecting the appropriate method.

\section{Conclusions}

The classification results obtained in this study indicate that image property covariance features provide a highly accurate solution for detection of popcorn kernels infected by the blue-eye fungus. The recognition rates that can be achieved using covariance-based features are superior for reflectance- and transmittance-mode images compared with previous methods. For both modes, overall recognition rates of more than $94 \%$ can be achieved by selecting different classification methods defined with different property vectors. This finding is important because the low recognition rates for reflectance mode images was an important problem, which, from an optics point of view, are easier to acquire. Higher recognition rates for transmittance mode images are currently of little practical value because it is difficult to acquire transmittance images for real-time applications. Additionally, the recognition rates of undamaged and damaged kernel images were similar in this study. The results indicate that detection of blue-eye damaged popcorn is possible with sufficient accuracy to be of use to popcorn processors. Future study will focus on real-time implementation of the algorithm.

\section{Acknowledgments}

This paper reports the results of research only. Any mention of a proprietary product or trade name does not constitute a recommendation or endorsement by the US Department of Agriculture.

USDA is an equal opportunity provider and employer.

\section{References}

Boser, B.-E., Guyon, I.-M., Vapnik, V.-N., 1992. A training algorithm for optimal margin classifiers. In: Proceedings of the Fifth Annual Workshop on Computational Learning Theory (COLT). ACM, New York, NY, USA, pp. 144152. URL http://doi.acm.org/10.1145/130385.130401.

Cakir, S., Cetin, A.-E., 2011. Mel- and mellin-cepstral feature extraction algorithms for face recognition. The Computer Journal 54 (9), 1526-1534. URL http:// comjnl.oxfordjournals.org/content/54/9/1526.abstract.

Cetin, A.-E., Pearson, T.-C., Tewfik, A.-H., 2004. Classification of closed- and openshell pistachio nuts using voice recognition technology. Transactions of the ASABE 47 (2), 659-664.

Chang, C.-C., Lin, C.-J., 2011. Libsvm: A library for support vector machines. ACM Transactions of Intelligent System Technologies 2, 1-27. URL http://doi.acm.org/ 10.1145/1961189.1961199.

Duman, K., Cetin, A.-E., 2010. Target detection in sar images using code difference and directional filters. In: Zelnio, E.G., Garber, F.D. (Eds.), Algorithms for 3 Synthetic Aperture Radar Imagery XVII. Vol. 7699. SPIE. URL http://link.aip.org/ link/?PSI/7699/76990S/1.

Duman, K., Eryildirim, A., Cetin, A.-E., 2009. Target detection and classification in sar images using region covariance and co-difference. In: Zelnio, E.G., Garber, F.D. (Eds.), Algorithms for Synthetic Aperture Radar Imagery XVI. Vol. 7337. SPIE. URL http://link.aip.org/link/?PSI/7337/73370P/1.

Eryildirim, A., Onaran, I., 2011. Pulse doppler radar target recognition using a twostage svm procedure. Aerospace and Electronic Systems, IEEE Transactions 47 (2), 1450-1457.

Forstner, W., Moonen, B., Gauss, C.-F., 1999. A metric for covariance matrices. Technical Report, Dept. of Geodesy and Geoinformatics, Stuttgard University, Germany.

Habiboglu, Y.-H., Gunay, O., Cetin, A.-E., 2011a. Covariance matrix-based fire and flame detection method in video. Machine Vision and Applications 22, 1-11.

Habiboglu, Y.-H., Gunay, O., Cetin, A.-E., 2011b. Real-time wildfire detection using correlation descriptors. European Signal Processing Conference, Barcelona, Spain.

Narwaria, M., Lin, W., Cetin, A.-E., 2012. Scalable image quality assessment with 2d mel-cepstrum and machine learning approach. Pattern Recognition 45 (1), 299313. URL http://www.sciencedirect.com/science/article/pii/S0031320311002962.

Pearson, T.-C., 2009. Hardware-based image processing for high-speed inspection of grains. Computers and Electronics in Agriculture 69 (1), 12-18. URL http:// www.sciencedirect.com/science/article/pii/S0168169909001021.

Pearson, T.-C., Cetin, A.-E., Tewfik, A.-H., Gokmen, V., 2007a. An overview of signal processing for food inspection [applications corner]. Signal Processing Magazine, IEEE 24 (3), 106-109.

Pearson, T.-C., Cetin, A.-E., Tewfik, A.-H., Haff, R.-P., 2007b. Feasibility of impactacoustic emissions for detection of damaged wheat kernels. Digital Signal Processing 17 (3), 617-633. URL http://www.sciencedirect.com/science/article/ pii/S1051200405001028.

Pearson, T.-C., Moore, D., Pearson, J.-E., 2011. A machine vision system for high speed sorting of small spots on grains. Submitted to Sensors and Instrumentation for Food Quality and Safety.

Quatieri, T.-F., Nov. 2001. Discrete-Time Speech Signal Processing: Principles and Practice. Prentice Hall. URL http://www.worldcat.org/isbn/013242942X.

Tuna, H., Onaran, I., Cetin, A.-E., 2009. Image description using a multiplier-less operator. IEEE Signal Processing Letters 16 (9), 751-753.

Tuzel, O., Porikli, F., Meer, P., 2006. Region covariance: A fast descriptor for detection and classification. In: Leonardis, A., Bischof, H., Pinz, A. (Eds.), Proc. of European Conference on Computer Vision (ECCV). Vol. 3952 of Lecture Notes in Computer Science. Springer Berlin/Heidelberg, pp. 589-600.

Yorulmaz, O., Pearson, T. C., Cetin, A. E., 2011. Cepstrum based feature extraction method for fungus detection. Vol. 8027. SPIE, p. 80270E. URL http://link.aip.org/ link/?PSI/8027/80270E/1. 